\title{
Dimension Formula for Random Transformations ${ }^{\star}$
}

\author{
F. Ledrappier and L.-S. Young $\star \star$ \\ Laboratoire de Probabilités, 4 Place Jussieu, Tour 56, F-75230 Paris Cedex 05, France
}

\begin{abstract}
We consider compositions of random diffeomorphisms and show that the dimension of sample measures equals Lyapunov dimension as conjectured in the nonrandom case by Yorke et al.
\end{abstract}

The role of dimension has long been recognized in the study of chaos (see [ER]). Roughly speaking, the dimension of a set measures the amount of information necessary to specify points within it accurately. In practice it tells us how many variables we should use to parametrize the set [Man]. This information is particularly useful for dealing with attractors of relatively low dimension embedded in a high, possibly infinite, dimensional phase space.

Various methods of experimentally computing dimension have been devised. A widely used procedure is to estimate dimension via Lyapunov exponents, computing a quantity called Lyapunov dimension introduced by Yorke et al [FKYY]. The validity of this procedure relies on Yorke's conjecture, a version of which states that "typically" Lyapunov dimension equals the usual notions of dimension. This is true for attractors on surfaces [Y] but when the phase space has dimension greater than 2, Yorke's conjecture has not been mathematically verified.

In this paper we do not deal with the conjecture in [FKYY] exactly as it is stated. Instead, we assume that our dynamical system is subjected to certain types of stochastic noise. We prove under this assumption that Yorke's conjectured formula for dimension is indeed mathematically correct. The model we use consists of composing random diffeomorphisms with some conditions to guarantee genuine randomness. We prove that in this setting Lyapunov dimension is equal to the dimension of the sample measures, i.e. the natural invariant family of measures associated with individual realizations of the random process. Our results are applicable to flows arising from stochastic differential equations.

\section{Introduction}

Consider first the nonrandom case. Let $f: M \bigcirc$ be a diffeomorphism of a compact Riemannian manifold preserving an ergodic Borel probability measure

* Both authors are supported by AFOSR. The second author is also supported by NSF and the Sloan Foundation

$\star \star$ Permanent address: Department of Mathematics, University of Arizona, Tucson, AZ 85721, USA 
$m$, and let $\lambda_{1}>\cdots>\lambda_{r}$ denote its distinct Lyapunov exponents. The authors of [FKYY] conjecture that if $f$ has an attractor and $m$ is its Sinai-Bowen-Ruelle measure, then, pathological cases excepted, the capacity of the attractor is given by a quantity they call the Lyapunov dimension of the attractor. This quantity is an expression involving the $\lambda_{j}$ 's and their respective multiplicities. We shall denote it here by $\mathscr{D}\left(\lambda_{1}, \ldots, \lambda_{r}\right)$ and refer the reader to Sect. 1 for its explicit definition.

Later on, with $\operatorname{dim}(m)$ denoting the dimension of the measure $m$, we show in [LY1] (see also $[\mathrm{T}]$ ) that for every ergodic invariant probability measure $m$,

$$
\overline{\operatorname{dim}}(m) \leqq \mathscr{D}\left(\lambda_{1}, \ldots, \lambda_{r}\right)^{1} .
$$

In fact, we show that in order for $\overline{\operatorname{dim}} m$ to equal $\mathscr{D}\left(\lambda_{1}, \ldots, \lambda_{r}\right)$, the partial dimensions of $m$ must assume a special configuration: $m$ must take on maximal dimension in the directions corresponding to $\lambda_{1}, \ldots, \lambda_{j}$ before "spilling over" into the $\lambda_{j+1}$ direction. In particular, $m$ must be a Sinai-Bowen-Ruelle measure.

We do not know how prevalent SBR measures are, or if this dimensional configuration is generic among diffeomorphisms preserving SBR measures. We will show however that the above configuration is a likely scenario for stochastically perturbed dynamical systems.

More precisely, let $v$ be a probability measure on $\operatorname{Diff}(M)$, the space of diffeomorphisms of $M$. We consider the composition of maps chosen independently with distribution $v$. This process together with an ergodic stationary measure $\mu$ will be denoted $\mathscr{X}=\mathscr{X}(M, v ; \mu)$. Let $\left\{\mu_{\omega}, \underline{\omega} \in \operatorname{Diff}(M)^{\mathbb{Z}}\right\}$ be its associate family of sample measures. That is, if $\underline{\omega}=\left\{f_{n}\right\}_{n \in Z}$, then $\mu_{\omega}$ gives the conditional distribution on $M$ at time 0 given that $\left\{f_{n}\right\}_{n<0}$ has been applied. Recall that Lyapunov exponents for $\mathscr{X}$ are well defined and are nonrandom. We continue to denote them by $\lambda_{1}>\cdots>\lambda_{r}$.

In [LY2] we proved an entropy formula in this random diffeomorphism setting. To prove this entropy formula, it is sufficient to assume that the transition probabilities of $\mathscr{X}$ have densities, so that the image of each point is smeared. Here we ask for a little bit more. We need to assume that the process randomizes the relative positions of all pairs of nearby points. This can be formulated in various ways; see hypotheses $A, A^{\prime}$ and $B$ in Sect. 1 .

Main Result. Suppose $\mathscr{X}(M, v ; \mu)$ satisfies Hypothesis $A, A^{\prime}$ or $B$, and $\lambda_{j} \neq 0 \forall j$. Then, for $v^{\mathbb{Z}}$. a.e. $\underline{\omega} \in \operatorname{Diff}(M)^{\mathbb{Z}}$,

$$
\operatorname{dim}\left(\mu_{\underline{\omega}}\right)=\mathscr{D}\left(\lambda_{1}, \ldots, \lambda_{r}\right)
$$

The proofs of this and other results are carried out in Sects. 3 and 4. In Sect. 5, we attempt to verify some of our hypotheses for stochastic flows.

\section{Definitions and Statements of Results}

(1.1) Setting. The general setting of this paper is identical to that in [LY2], as are

\footnotetext{
${ }^{1}$ The limit in the definition of $\operatorname{dim}(m)$ does not always exist. We actually prove this inequality using lim sup
} 
all the basic notations. We recall them for the convenience of the reader. A more detailed discussion is given in [LY2].

It is assumed throughout that $M$ is a compact Riemannian manifold, $\operatorname{Diff}^{2}(M)$ is the group of $C^{2}$ diffeomorphisms of $M$ onto itself, and $(\Omega, \mathscr{F})$ is a probability space identified with $\operatorname{Diff}^{2}(M)$ together with its natural Borel $\sigma$-algebra. For $\omega \in \Omega$, we denote the corresponding diffeomorphism by $f_{\omega}$. Let $v$ be a probability measure on $(\omega, \mathscr{F})$ satisfying

$$
\begin{array}{r}
\int \log ^{+}\left|f_{\omega}\right|_{C^{2}} v(d \omega)<+\infty, \\
\int \log ^{+}\left|f_{\omega}^{-1}\right|_{C^{2}} v(d \omega)<+\infty .
\end{array}
$$

We consider the composition of $\left\{f_{\omega_{n}}\right\}_{n=-\infty}^{\infty}$, where the $\omega_{n}$ 's are chosen independently with distribution $v$. Let $\mu$ be an ergodic stationary measure for this process, i.e. $\mu$ satisfies

$$
\mu=\int f_{\omega} \mu v(d \omega)
$$

and is extremal with respect to this property. In the rest of this paper, this stationary process will be referred to as $\mathscr{X}(M, v ; \mu)$ or simply $\mathscr{X}$.

Associated with $\mathscr{X}(M, v ; \mu)$ is a canonical family of sample measures $\left\{\mu_{\omega}, \underline{\omega} \in \Omega^{\mathbb{Z}}\right\}$. This family is the unique measurable family of probabilities on $M$ satisfying

and

i) $f_{\omega_{0}} \mu_{\underline{\omega}}=\mu_{\tau \underline{\omega}}, \quad$ where $(\underline{\omega})_{n}=\omega_{n}, \quad(\tau \underline{\omega})_{n}=\omega_{n+1}$,

ii) $\underline{\omega} \rightarrow \mu_{\omega}$ depends only on $\omega_{n}$ for $n<0$,

iii) $\int \mu_{\underline{\omega}} v^{\mathbb{Z}}(d \underline{\omega})=\mu$.

Most of our arguments are carried out in the context of the skew product representation of $\mathscr{X}$, which is given by the measure preserving transformation $F:\left(\Omega^{\mathbb{Z}} \times M\right)$, with

and

$$
F(\underline{\omega}, x)=\left(\tau \underline{\omega}, f_{\omega_{0}} x\right)
$$

$$
\mu^{*}(d \underline{\omega}, d x)=v^{\mathscr{Z}}(d \underline{\omega}) \mu_{\underline{\omega}}(d x) .
$$

(1.2) Definition of Key Words. For $\underline{\omega \in} \Omega^{\mathbb{Z}}$ and $n \geqq 0$, let

and

$$
f_{\underline{\omega}}^{n}=f_{\omega_{n-1}} \circ \cdots \circ f_{\omega_{0}}
$$

$$
f_{\underline{\omega}}^{-n}=f_{\omega_{n-1}}^{-1} \circ \cdots \circ f_{\omega_{n-1}}^{-1} .
$$

The numbers $\lambda_{1}>\cdots>\lambda_{r}$ with multiplicities $m_{1}, \ldots, m_{r}$ respectively are called the Lyapunov exponents of $\mathscr{X}$ if for $\mu^{*}$-a.e. $(\underline{\omega}, x)$ there is a splitting

$$
T_{x} M=E_{1}(\underline{\omega}, x) \otimes \cdots \otimes E_{r}(\underline{\omega}, x)
$$

such that $\operatorname{dim} E_{j}(\underline{\omega}, x)=m_{j}$ and $v \neq 0 \in E_{j}(\underline{\omega}, x)$ if and only if

and

$$
\lim _{n \rightarrow \infty} \frac{1}{n} \log \left|D\left(f_{(1)}^{n}\right)_{x} v\right|=\lambda_{j}
$$

$$
\lim _{n \rightarrow \infty} \frac{1}{n} \log \left|D\left(f_{\underline{\omega}}^{-n}\right)_{x} v\right|=-\lambda_{j}
$$


The integrability condition in (1.1) and the ergodicity of $\mu$ guarantee that these numbers exist. We will always assume that $\lambda_{1}>0$, for otherwise our statements hold rather trivially.

Let $E^{j}=\bigoplus_{i \geq 1} E_{i}$. For $j$ with $\lambda_{j}<0$, we define the stable manifold corresponding to $E^{j}$ at $(\underline{\omega}, x)$ to be

$$
W^{j}(\underline{\omega}, x)=\left\{y \in M: \limsup _{n \rightarrow \infty} \frac{1}{n} \log d\left(f_{\underline{\underline{\omega}}}^{n} x, f_{\underline{\underline{\omega}}}^{n} y\right) \leqq \lambda_{j}\right\} .
$$

The Lyapunov dimension of $\mathscr{X}$ is defined exactly as in the non-random case (as introduced in [FKYY]). Let $K$ be the largest integer so that $\sum_{j=1}^{K} \lambda_{j} m_{j}>0$. Then the Lyapunov dimension of $\mathscr{X}$ is defined to be

$$
\mathscr{D}\left(\lambda_{1}, \ldots, \lambda_{r}\right)=\left\{\begin{array}{l}
\operatorname{dim} M \quad \text { if } \sum_{j=1}^{K} m_{j}=\operatorname{dim} M \\
\sum_{j=1}^{K} m_{j}-\frac{1}{\lambda_{K+1}} \sum_{j=1}^{K} \lambda_{j} m_{j} \quad \text { otherwise. }
\end{array}\right.
$$

We use $\mathscr{D}\left(\lambda_{1}, \ldots, \lambda_{r}\right)$ to denote Lyapunov dimension to stress the fact that despite the terminology, Lyapunov dimension in general does not necessarily have the geometric properties of a dimension.

Finally, the dimension of a finite Borel measure $m$ on a compact metric space is defined to be $\sigma$, written $\operatorname{dim}(m)=\sigma$, if

$$
\lim _{\varepsilon \rightarrow 0} \frac{\log m B(x, \varepsilon)}{\log \varepsilon}=\sigma
$$

for $m$-a.e.x. (Here, as in the rest of the paper, $B(x, \varepsilon)$ is the ball of radius $\varepsilon$ about $x$.) We are interested in the dimension of the $\mu_{\omega}$ 's. Clearly, if $\operatorname{dim}\left(\mu_{\omega}\right)$ is well defined for a.e. $\underline{\omega}$, then $\underline{\omega} \rightarrow \operatorname{dim}\left(\mu_{\underline{\omega}}\right)$ is constant $v^{\mathscr{E}}$ a.e.

Throughout this paper, whenever $X$ is understood to have a natural Riemannian volume, we use the term "Lebesgue measure on $X$ " to denote the measure associated with this Riemannian volume.

As mentioned in the introduction, there are various ways of formulating the hypotheses that would give the desired result. We state a few versions in the next two subsections.

(1.3) Version $A$ of the Result. Let $L$ be the smallest integer so that $\sum_{j=1}^{L} \lambda_{j} m_{j} \leqq 0$ (i.e. $L=K+1$ for $K$ in (1.2)).

Recall that $E^{j}=\bigoplus_{i \geq j} E_{i}$.

Hypothesis $A$. For $\mu$-a.e.x and $j=L, L+1$, the distribution of $\underline{\omega} \rightarrow E^{j}(\underline{\omega}, x)$ is absolutely continuous with respect to Lebesgue on the space of $\left(\sum_{i \geqq j} m_{i}\right)$-planes in $T_{x} M$. 
Theorem A. Let $\mathscr{X}(M, v ; \mu)$ be so that the measure $\mu$ is absolutely continuous with respect to Lebesgue on $M$ and $\lambda_{j} \neq 0$ for all $j$. Assume Hypothesis $A$ is satisfied. Then, for $v^{\mathscr{Z}}-$ a.e. $\underline{\omega}$,

$$
\operatorname{dim}\left(\mu_{\underline{\omega}}\right)=\mathscr{D}\left(\lambda_{1}, \ldots, \lambda_{r}\right)
$$

Theorem A is proved in Sect. 4.

We mention also a stronger set of hypotheses that appears quite naturally. Recall that the Grassmannian bundle of $M$ is:

$$
\operatorname{Gr}(M)=\sum_{k=1}^{\operatorname{dim} M} \operatorname{Gr}(M, k)
$$

where $\operatorname{Gr}(M, k)$ is the bundle of $k$-dimensional subspaces of tangent spaces to $M$. We define the "backward derivative process" associated with $\mathscr{X}$ as follows: for $v \in \operatorname{Gr}(M)$ and $\Gamma \subset \operatorname{Gr}(M)$, the probability transition kernel $Q(v, \Gamma)$ is

$$
Q(v, \Gamma)=v\left\{\omega:\left(D f_{\omega}^{-1}\right) v \in \Gamma\right\}
$$

Hypothesis $A^{\prime}$. For all $v \in \operatorname{Gr}(M)$, the probability $Q(v, \cdot)$ is absolutely continuous with respect to Lebesgue on $\operatorname{Gr}(M)$.

Theorem A'. Theorem A continues to hold if Hypothesis $A$ is replaced by Hypothesis $A^{\prime}$.

In Sect. 5, we show why Hypothesis $\mathrm{A}^{\prime}$ implies Hypothesis A and we indicate why Hypothesis $\mathrm{A}^{\prime}$ is easy to verify for stochastic flows.

(1.4) Version B of the Result. In (1.3) we assume essentially that there is some diffusion on the derivative level. We now give a nonlinear version of that, formulated in terms of two-point processes. As we shall see, one advantage of this new formulation is that it yields absolutely continuous transverse measures.

Let $(x, z) \rightarrow v^{x, z}$ be a measurable family of probability measures on $\Omega$ with the property that for Lebesgue-a.e. fixed $x,\left\{v^{x, z}, z \in M\right\}$ is a family of conditional measures associated with the partition $P_{x}$ on $\Omega$ defined by $P_{x}(z)=\left\{\omega: f_{\omega} x=z\right\}$. For all $y \in M$, we let $P_{x, z}(y, \cdot)$ denote the image of the measure $v^{x, z}$ under the map $\omega \rightarrow f_{\omega} y$, and $\rho_{y}^{x, z}$ denote the density of $P_{x, z}(y, \cdot)$ if it exists.

Hypothesis $B$.

i) For Lebesgue-a.e. $(x, z)$ and all $y$ the measure $P_{x, z}(y, \cdot)$ is absolutely continuous with respect to Lebesgue on $M$.

ii) For all $\xi>0$, there exists $G_{\xi} \subset M \times M$ with Lebesgue $\left(M \times M \backslash G_{\xi}\right)=0$, and a measurable function $\mathscr{E}_{\xi}: G_{\xi} \rightarrow \mathscr{R}^{+}$such that for all $(x, z) \in G_{\xi}$ and all $y$ with $d(x, y) \leqq \mathscr{E}_{\xi}(x, z)$

$$
\rho_{y}^{x, z} \leqq d(x, y)^{-\operatorname{dim} M-\xi} .
$$

Observe that when Hypothesis B is satisfied, then for all $y$ the measure $P(y, \cdot)=: v\left\{\omega: f_{\omega} y \in \cdot\right\}$ is absolutely continuous with respect to Lebesgue on $M$. This can be seen by integrating condition i) over $z$. Also when i) is satisfied, condition ii) gives a scaling for the density $\rho_{y}^{x, z}$ in terms of $d(x, y)$. To see that this is in some sense a natural scaling, consider for instance a probability $\hat{v}$ on $G L(n, \mathscr{R})$ with a 
bounded density and estimate the density $\hat{\rho}_{y}$ of the image of $y \in \mathscr{R}^{n}$ under these random linear maps as $|y|$ goes to zero. See also [Le] for a class of stochastic flows for which Hypothesis B can be verified.

Theorem B. Let $\mathscr{X}(M, \omega ; \mu)$ be so that $\lambda_{j} \neq 0$ for all $j$ and Hypothesis $B$ is satisfied. Then, for a.e. $\underline{\omega}$

$$
\operatorname{dim}\left(\mu_{\underline{\omega}}\right)=\mathscr{D}\left(\lambda_{1}, \ldots, \lambda_{r}\right) .
$$

We now state the geometric property of transverse measures alluded to earlier. Let $j$ be such that $\lambda_{j}<0$ and let $d_{j}=\sum_{i<j} m_{i}=\operatorname{dim} M-\operatorname{dim} E^{j}$. Let $S \subset \mathscr{R}^{d_{j}}$ be a compact subset and let $D$ be the unit disk in $\mathscr{R}^{\operatorname{dim} E^{\jmath}}$. A mapping $\Phi: S \times D \rightarrow M_{\omega}=$ $\{\underline{\omega}\} \times M$ is said to define a continuous family of $W^{j}$-disks if it is a topological embedding and for each $s \in S,\left.\Phi\right|_{\{s\} \times D}$ maps diffeomorphically onto an open subset of $W^{j}\left(\underline{\omega}, x_{s}\right)$ for some $x_{s}$. We say that $\left\{\mu_{\underline{\omega}}\right\}$ has absolutely continuous transverse measures with respect to $W^{j}$ if for a.e. $\underline{\omega}$, whenever $\Phi$ is as above and $\mu_{\omega}(\Phi(S \times D))>0$, then $\mu_{\omega} \mid \Phi(S \times D)$ projected along these $W^{j}$-disks into any smooth transversal $\Sigma$ is a bsolutely continuous with respect to Lebesgue on $\Sigma$.

Theorem $\mathbf{B}^{\prime}$. Let $\mathscr{X}$ be so that $\lambda_{j} \neq 0$ for all $j$ and Hypothesis $B$ is satisfied. If $\lambda_{j}<0$ and $d_{j}<\operatorname{dim}\left(\mu_{\omega}\right)$, then $\left\{\mu_{\omega}\right\}$ has absolutely continuous transverse measures with respect to $W^{j}$.

Theorem B and Theorem B' are proved in Sect. 3.

\section{Preparations for the Proofs}

In this section, we recall some known facts from the smooth ergodic theory of random maps. These facts were first established in the nonrandom case, and have been observed to carry over easily (see e.g. [Ki, Ca, LY2]).

(2.1) Properties of Local Stable Manifolds. Let us agree to write $F^{j}(\underline{\omega}, x)=E^{j}(\underline{\omega}, x)^{\perp}$, the orthogonal complement of $E^{j}(\underline{\omega}, x)$ in $T_{x} M$. We fix $j$ with $\lambda_{j}<0$ for the rest of this subsection.

Let $\varepsilon>0$ be given. Then there are positive numbers $C_{0}, \alpha, D_{0}, \beta, E_{0}, \delta_{0}, \delta_{1}$ and a measurable set

$$
\Lambda=\Lambda\left(C_{0}, \alpha, D_{0}, \beta, E_{0}, \delta_{0}, \delta_{1}\right) \subset \Omega^{\mathscr{Z}} \times M,
$$

such that the following five properties hold:

(i) $\Lambda$ depends only on $x$ and $\omega_{n}, n \geqq 0$, and $\mu^{*} \Lambda \geqq 1-\varepsilon$.

(ii) For $(\underline{\omega}, x) \in \Lambda$ and $n \geqq 0$,

$$
\begin{aligned}
& v \in E^{j}(\underline{\omega}, x) \Rightarrow\left\|\left(D f_{\underline{\omega}}^{n}\right)_{x} v\right\| \leqq C_{0} e^{n\left(\lambda_{j}+\varepsilon\right)}\|v\|, \\
& v \in F^{j}(\underline{\omega}, x) \Rightarrow\left\|\left(D f_{\underline{\omega}}^{n}\right)_{x} v\right\| \geqq C_{0}^{-1} e^{n\left(\lambda_{j-1}-\varepsilon\right)}\|v\| .
\end{aligned}
$$

(iii) For each $(\underline{\omega}, x)$ in $\Lambda$, there is an embedded $\left(\sum_{i \geqq j} m_{i}\right)$-dimensional disk $W_{\alpha}^{j}(\underline{\omega}, x)$ such that

a) $W_{\alpha}^{j}(\underline{\omega}, x)=\left\{y \in W^{j}(\underline{\omega}, x): d^{j}(y, x) \leqq \alpha\right\}$, where $d^{j}$ is the metric along $W^{j}(\underline{\omega}, x)$, 
b) $\exp _{x}^{-1} W_{\alpha}^{j}(\underline{\omega}, x)$ is part of the graph of a function $g_{\underline{\omega}, x}: E^{j}(\underline{\omega}, x) \rightarrow F^{j}(\underline{\omega}, x)$ satisfying
i. $g_{\omega, x} 0=0$,
ii. $D g_{\omega, x}(0)=0$,
iii. $\left|D g_{\omega, x}\right| \leqq 1 / 1000$,
iv. $\operatorname{Lip}\left(D g_{\omega, x}\right) \leqq C_{0}$,

and

c) If $z_{1}, z_{2} \in W_{\alpha}^{j}(\underline{\omega}, x)$, then $d^{j}\left(f^{n} z_{1}, f^{n} z_{2}\right) \leqq C_{0} e^{n\left(\lambda_{j}+\varepsilon\right)} d^{j}\left(z_{1}, z_{2}\right)$ for all $n \geqq 0$.

(iv) Let $\Lambda_{\omega}=\{x \in M:(\underline{\omega}, x) \in \Lambda\}$. Then for each $\underline{\omega}$ with $\Lambda_{\omega}$ non-empty, the map $x \rightarrow E^{j}(\underline{\omega}, x)$ is locally Hölder continuous on the set $W_{\alpha}^{j}\left(\Lambda_{\underline{\omega}}^{-}\right)=: \bigcup_{x \in \Lambda_{\omega}} W_{\alpha}^{j}(\underline{\omega}, x)$.

In fact, for all $z_{1} z_{2} \in W_{\alpha}^{j}\left(\Lambda_{\omega}\right)$ with $d\left(z_{1}, z_{2}\right) \leqq \delta_{0}$,

$$
\operatorname{dist}\left(E^{j}\left(\underline{\omega}, z_{1}\right), E^{j}\left(\underline{\omega}, z_{2}\right)\right) \leqq D_{0} d\left(z_{1}, z_{2}\right)^{\beta} .
$$

[We have this estimate on the entire set $W_{\alpha}^{j}\left(\Lambda_{\omega}\right)$ (as opposed to just $\Lambda_{\omega}$ ) because by virtue of (iii)c), property (ii) holds for all points in $W_{\alpha}^{j}\left(\Lambda_{\omega}\right)$ with a slightly weaker constant.]

Property (iv) follows from Proposition 5.4 of [BK]. We remark that since we do not have uniform bounds of $D f$ and $D^{2} f$, the Hölder constant and the Hölder exponent depend on $\underline{\omega}$. The constant $a$ in [BK] must be replaced by $a(\underline{\omega})=\sup _{n}(1 / n) \log \left|f_{\underline{\omega}}^{n}\right|_{c^{2}}$.

Finally:

(v) Let $\underline{\omega}$ be such that $\Lambda_{\omega}$ is non-empty. For $x \in \Lambda_{\omega}$, let $T_{1}$ and $T_{2}$ be $\exp _{x}$-images of small disks parallel to $F^{j}(\underline{\omega}, x)$ and at a distance smaller than $\delta_{1}$ from $F^{j}(\underline{\omega}, x)$. Then the map $\psi$ from $T_{1} \cap W_{\alpha}^{j}\left(\Lambda_{\omega} \cap B\left(x, \delta_{0}\right)\right)$ to $T_{2}$ by sliding along $W_{\alpha}^{j}$-leaves is absolutely continuous with $|\operatorname{Jac}(\bar{\psi})| \leqq E_{0}$.

See $[\mathrm{BN}]$ or $[\mathrm{KS}]$ Chap II.12 for a proof of this absolute continuity property for the iteration of one map using only positive iterates. The proof here for each fixed sequence $\underline{\omega}$ is identical to that for one map; once again, some of the "constants" now depend on $\underline{\omega}$.

(2.2) Dimension Properties. We recall the definition of "partial dimensions" for $\left\{\mu_{\omega}\right\}$. These are numbers $\gamma_{i}, i=1, \ldots, r$, with the following properties:

(i) $0 \leqq \gamma_{i} \leqq m_{i}$ for $i=1, \ldots, r$,

(ii) $\gamma_{i_{0}}=m_{i_{0}}$ if $\lambda_{i_{0}}=0$,

(iii) $\sum_{i=1}^{r} \lambda_{i} \gamma_{i}=0$,

(iv) $\limsup _{\varepsilon \rightarrow 0} \frac{\log \mu_{\omega} B(x, \varepsilon)}{\log \omega} \leqq \sum_{i=1}^{r} \gamma_{i}$

for $\mu^{*}$ - a.e. $(\underline{\omega}, x)$. (This number will be denoted $\bar{\sigma} ; \underline{\sigma}$ is defined analogously using 
$\lim$ inf. When $\underline{\sigma}=\bar{\sigma}$ we write $\sigma$.)

(v) If $\lambda_{j}<0$, then $\sum_{i \geqq j} \gamma_{i}$ is the dimension

of the conditional measure of $\left\{\mu_{\underline{\omega}}\right\}$ on $W^{j}$; an analogous statement holds for $\sum_{i \leqq j} \gamma_{i}$ when $\lambda_{j}>0$.

The precise meaning of the "dimension of conditional measures on $W^{j}$ " as defined in [LY1] is as follows. (One could also say this in terms of continuous families of $W^{j}$-disks as in (1.4).) Let $\xi$ be a measurable partition on $\Omega^{\mathscr{Z}} \times M$ subordinate to $W^{j}$, that is, if $\xi_{\omega}$ denotes the restriction of $\xi$ to $\{\underline{\omega}\} \times M$, then for a.e. $(\underline{\omega}, x), \xi_{\underline{\omega}}(x)$ contains an open neighborhood of $x$ in $W^{j}(\underline{\omega}, x)$. Let $\left\{\mu_{\underline{\omega}}^{\xi}(x)\right\}$ be a canonical family of conditional measures of $\mu_{\omega}$ associated with $\xi_{\omega}$. Let $B_{\underline{\omega}}^{j}(x, \varepsilon)=\left\{y \in W^{j}(\underline{\omega}, x): d^{j}(x, y)<\varepsilon\right\}$. Then property $(\mathrm{v})$ says that for $\mu^{*}-$ a.e. $(\underline{\omega}, x)$,

$$
\lim _{\varepsilon \rightarrow 0} \frac{\log \mu_{\omega}^{\xi} B_{\omega}^{j}(x, \varepsilon)}{\log \varepsilon}=\sum_{i \geqq J} \gamma_{i} .
$$

See [LY1] for the non-random version of the results stated here. The proofs in the random case are parallel, as mentioned in [LY2].

Suppose now that the stationary measure $\mu$ is absolutely continuous with respect to Lebesgue on $M$. Then Pesin's entropy formula holds, or equivalently, $\left\{\mu_{\omega}\right\}$ has absolutely continuous conditional measures on unstable manifolds [LY2]. Then, if $\mu$ is ergodic and $\lambda_{j} \neq 0$ for all $j$, we have

$$
\bar{\sigma}=\underline{\sigma}=\sum_{i} \gamma_{i} \quad(\text { see }[\mathrm{LY} 2])
$$

Observe that these conditions are satisfied under the hypotheses of Theorems A, $\mathrm{A}^{\prime}, \mathrm{B}$ and $\mathrm{B}^{\prime}$.

Finally we mention the relation between Lyapunov dimension and the $\gamma_{j}$ 's. It is easy to check (using property (iii) above) that $\sum_{i=1}^{r} \gamma_{i} \leqq \mathscr{D}\left(\lambda_{1}, \ldots, \lambda_{r}\right)$ always, and that if $\sum_{i=1}^{r} \lambda_{i} m_{i} \leqq 0$-which is always the case for $\mathscr{X}(M, v ; \mu)$ - then equality is attained if and only if there is a critical number $j$, denoted $j_{c}$, so that

and

$$
\gamma_{j}=\left\{\begin{array}{lll}
m_{j} & \text { for } & j<j_{c} \\
0 & \text { for } & j>j_{c}
\end{array}\right.
$$

$$
0<\gamma_{j_{c}} \leqq m_{j_{c}} .
$$

Thus under the hypotheses of our theorems, this dimensional configuration is both necessary and sufficient for the desired dimension formula

$$
\sigma=\mathscr{D}\left(\lambda_{1}, \ldots, \lambda_{r}\right)
$$

\section{Proof of Version B of the Results}

In (3.1) we sketch the main ideas in the proof of Theorem B. The statements in this subsection need not be technically accurate. The formal proof is carried out in 
(3.2) and (3.3), and Theorem $\mathrm{B}^{\prime}$ is proved in (3.4).

(3.1) Strategy of Proof of Theorem B. Let $j$ be such that $\lambda_{j}<0$. First we attempt to define a notion of "transverse dimension" for $\mu_{\omega}$ with respect to $W^{j}$. That is, we project $\mu_{\omega}$ along $W_{\alpha}^{j}$-leaves onto a transversal in $M$ and call its dimension $\tau_{j}$. (Actually we do not show that this transverse dimension exists, but ignore this for now.) Recall that $d_{j}=\operatorname{dim} F^{j}$ and $\sigma=\operatorname{dim}\left(\mu_{\omega}\right)$, which under the hypothesis of Theorem B is well defined and equals $\sum_{i} \gamma_{i}$. The main ingredient of the proof is to show that

$$
\tau_{j} \geqq \min \left(\sigma, d_{j}\right)
$$

The proof of this assertion relies heavily on Hypothesis B and is influenced by the ideas in [Mar]. There is another inequality which is a simple and general fact about adding the dimensions of fiber and transverse measures, namely,

$$
\sigma \geqq \tau_{j}+\sum_{i \geqq j} \gamma_{i}
$$

We now argue how $(*)$ combined with $(* *)$ give the desired result. Consider $j=r, r-1, \ldots$, in descending order. Initially we may have $\sigma \leqq d_{j}$ (or we may not). Suppose $\sigma \leqq d_{j}$. Then $(*)$ and $(* *)$ together imply that $\gamma_{i}=0$ for all $i \geqq j$. Let $L$ be the first $j$ so that $\sigma>d_{j}$, so in particular $\gamma_{i}=0$ for all $i>L$. We observe immediately that $\lambda_{L}<0$, otherwise $\gamma_{i}=0$ for all $i$ with $\lambda_{i}<0$, which is impossible since

$$
\sum_{\lambda_{i}<0}\left(-\lambda_{i}\right) \gamma_{i}=\sum_{\lambda_{i}>0} \lambda_{i} \gamma_{i}=\sum_{\lambda_{i}>0} \lambda_{i} m_{i}>0 .
$$

Now $(*)$ and $(* *)$ tell us that

$$
\sigma \geqq d_{L}+\sum_{i \geqq L} \gamma_{i}
$$

which together with the fact that $\sigma=\sum_{i} \gamma_{i}$ imply that $\gamma_{i}=m_{i}$ for all $i<L$. Thus the $\gamma_{j}$ 's assume the dimensional configuration described at the end of (2.2) with $j_{c}=L$, and hence

$$
\sigma=\mathscr{D}\left(\lambda_{1}, \ldots, \lambda_{r}\right)
$$

(3.2) Proof of Theorem B. We now formally carry out the steps outlined in (3.1). As before we fix $j$ with $\lambda_{j}<0$.

Let $\xi>0$ be an arbitrarily small constant. We choose $\delta$ and $C>0$ with $\delta \leqq \delta_{0}, \delta_{1}, \alpha / 100$. (See (2.1) for the definitions of $\delta_{0}, \delta_{1}$ and $\alpha$ ). Let

$$
\begin{aligned}
& \Gamma=F^{-1} \Lambda \cap\{(\underline{\omega}, x) \in \Lambda: \text { i) }\left|D f_{\omega_{0}}\right| \leqq C, \\
& \text { ii) } \mu_{\underline{\omega}} B(x, r) \leqq C r^{\underline{\underline{\sigma}}-\xi} \quad \forall r \leqq \delta, \\
&\text { and iii) } \left.\left(x, f_{\omega_{0}} x\right) \in G_{\xi} \text { and } \mathscr{E}_{\xi}\left(x, f_{\omega_{0}} x\right) \geqq \delta / C\right\} .
\end{aligned}
$$

The definitions of $G_{\xi}$ and $\mathscr{E}_{\xi}$ are given in (1.4). By choosing $\delta$ sufficiently small and $C$ sufficiently large, we may assume that $\mu^{*} \Gamma>0$.

For $(\underline{\omega}, x)$ in $\Gamma$, we let $\widehat{T}_{\omega, x}=F_{\omega_{0}}^{-1}\left(\exp _{f_{\omega}^{x}} F^{j}(F(\underline{\omega}, x))\right)$. (This local transversal is chosen mostly for convenience.) Let $\hat{\pi}_{\underline{\omega}, x}$ denote the projection along $W_{\alpha}^{j}$-leaves 
onto $\hat{T}_{\underline{\omega}, x}$. For $r>0$ we are interested in

$$
\mu^{\hat{T}}(\underline{\omega}, x, r)=: \mu_{\underline{\omega}}\left\{y \in \Gamma_{\underline{\omega}}: d(x, y) \leqq \delta / C \text { and } d^{\hat{T}}\left(\hat{\pi}_{\underline{\omega}, x} y, x\right) \leqq r\right\},
$$

where $d^{\hat{T}}$ is the distance along $\hat{T}_{\underline{\omega}, x}$. Let

$$
\tau_{j}(\underline{\omega}, x)=\limsup _{r \rightarrow 0} \frac{\log \mu^{\hat{T}}(\underline{\omega}, x, r)}{\log r} .
$$

Proposition 3.2.1 (Main Proposition). Assume Hypothesis B. Then the following is true for $\mu^{*}-$ a.e. $(\underline{\omega}, x)$ in $\Gamma$ :

(1) If $\underline{\sigma}-2 \xi<d_{j}$, then

$$
\liminf _{r \rightarrow 0} \frac{\mu^{\hat{T}}(\underline{\omega}, x, r)}{r^{\underline{\sigma}-2 \xi}}<\infty,
$$

so that in particular, $\tau_{j}(\underline{\omega}, x) \geqq \underline{\sigma}-2 \xi$.

(2) If $\underline{\sigma}-2 \xi>d_{j}$, then

$$
\liminf _{r \rightarrow 0} \frac{\mu^{\hat{T}}(\underline{\omega}, x, r)}{r^{d_{J}}}<\infty,
$$

so that in particular $\tau_{j}(\underline{\omega}, x) \geqq d_{j}$.

The proof of this proposition is postponed to (3.3).

We now shrink $\Gamma$ further to $\Gamma^{\prime}$ so that points in $\Gamma^{\prime}$ have the correct dimensional estimates along $W^{j}$. Let $\eta$ be a measurable partition of $\Omega^{\mathscr{Z}} \times M$ subordinate to $W^{j}$, and let $\left\{\mu_{\omega}^{\eta}(x)\right\}$ denote a family of conditional probability measures associated with $\eta$. Let $\delta^{\prime}>0$ be fixed.

We define

$$
\begin{gathered}
\Gamma^{\prime}=\left\{(\underline{\omega}, y) \in T: W_{\delta^{\prime}}^{j}(\underline{\omega}, y) \subset \eta(\underline{\omega}, y) \subset W_{\delta / 2 C}^{j}(\underline{\omega}, y)\right. \\
\text { and } \left.\mu_{\underline{\omega}}^{\eta}(y) B_{\underline{\omega}}^{j}(y, r) \leqq C r{ }_{l \geqq j}^{\left(\sum_{l} \gamma_{i}-\xi\right)} \text { for all } r \leqq \delta^{\prime}\right\} .
\end{gathered}
$$

By first choosing the partition $\eta$ so that most points satisfy $\eta(\underline{\omega}, y) \subset W_{\delta / 2 C}^{j}(\underline{\omega}, y)$, and then choosing $\delta^{\prime}$ sufficiently small, we can arrange to have $\mu^{*} \Gamma^{\prime}>0$.

Proposition 3.2.2. For $\mu^{*}$ - a.e. $(\underline{\omega}, x) \in \Gamma^{\prime}$,

$$
\bar{\sigma} \geqq \tau_{j}(\underline{\omega}, x)+\sum_{i \geqq j} \gamma_{i}-\xi
$$

Proof. For sufficiently small $r$,

$$
\mu_{\underline{\omega}} B(x, r) \leqq 2 \mu_{\underline{\omega}}\left(\Gamma_{\underline{\omega}}^{\prime} \cap B(x, r)\right)
$$

and since $y \in \Gamma_{\underline{\omega}}^{\prime} \cap B(x, r) \Rightarrow \eta(\underline{\omega}, y) \subset W_{\alpha}^{j}(\underline{\omega}, y) \cap B(x, \delta / C)$, we have

$$
\mu_{\underline{\omega}}\left(\Gamma_{\underline{\omega}}^{\prime} \cap B(x, r)\right) \leqq \mu^{\hat{T}}(\omega, x, N r) C(2 r)^{\sum_{i \geqq j}\left(\gamma_{i}-\xi\right)},
$$


where $N$ is a constant depending on the angles between $\hat{T}_{\omega, x}$ and the relevant $W^{j}$-leaves near $x$, which are uniformly bounded for $(\underline{\omega}, x)$ in $\bar{\Gamma}$. /

We remark that our proof so far does not depend on the fact that $\lambda_{i} \neq 0$ for all $i$. The no zero exponent hypothesis is used only to guarantee that

$$
\bar{\sigma}=\underline{\sigma}=\sum_{i} \gamma_{i} .
$$

Assuming that, we now run through the arguments in the last paragraph of (3.1). The reasoning there is valid since $\xi$ can be made arbitrarily small.

(3.3) Proof of the Main Proposition. Let $v^{x, z}$ be as defined in (1.4). Our strategy is to fix $\left\{\omega_{n}, n \neq 0\right\}, x$ and $z$, and to obtain an upper estimate for $\mu^{\hat{T}}(\underline{\omega}, x, r)$ for $v^{\boldsymbol{x}, z}$-a.e. $\omega$, where $(\underline{\omega}, x)=\left(\cdots \omega_{-2} \omega_{-1} \omega \omega_{1} \omega_{2} \ldots, x\right) \in \Gamma$. As $\omega$ varies we will always use $\underline{\omega}$ to denote the sequence with $(\underline{\omega})_{0}=\omega$ and fixed $(\underline{\omega})_{n}=\omega_{n}$ for $\mathrm{n} \neq 0$. Note that fixing $\omega_{n}$ for $n<0$ determines a sample measure which we denote by $\mu_{\omega}$. Our analysis will apply to $v^{\mathscr{Z} \backslash\{0\}}$-a.e. $\cdots \omega_{-2} \omega_{-1} \omega_{1} \omega_{2} \ldots, \mu_{\omega}$-a.e. $x$ and Lebesgue-a.e. $z$.

So let $\left\{\omega_{n}, n \neq 0\right\}, x$ and $z$ be fixed for the rest of the proof and define

$$
a_{r}=\int_{\{\omega:(\underline{\omega}, x) \in \Gamma\}} \mu^{\hat{T}}(\underline{\omega}, x, r) d v^{x, z}(\omega)
$$

We will show that

$$
\left\{\begin{array}{l}
\limsup _{r \rightarrow 0} \frac{a_{r}}{r^{-2 \xi}}<+\infty \text { if } \quad \underline{\sigma}-2 \xi<d_{j} \\
\limsup _{n \rightarrow 0} \frac{a_{r}}{r^{d_{J}}}<+\infty \text { if } \quad \underline{\sigma}-2 \xi>d_{j}
\end{array} .\right.
$$

This, together with Fatou's lemma gives the statement in Proposition 3.2.1. Now,

$$
\begin{aligned}
a_{r} & =\int_{\{\omega:(\underline{\omega}, x) \in \Gamma\}} \mu_{\underline{\omega}}\left\{y \in \Gamma_{\underline{\omega}}: d(x, y)<\delta / C \text { and } d^{\hat{T}}\left(\hat{\pi}_{\underline{\omega}, x} y, x\right) \leqq r\right\} d v^{x, z}(\omega) \\
& =\int_{B(x, \delta / C)} v^{x, z}\left\{\omega:(\underline{\omega}, x),(\underline{\omega}, y) \in \Gamma, d^{\hat{T}}\left(\hat{\pi}_{\underline{\omega}, x} y, x\right) \leqq r\right\} d \mu_{\underline{\omega}}(y) .
\end{aligned}
$$

We will estimate this integrand by working in a neighborhood of $z$. Observes first that whether $(\tau \underline{\omega}, z)$ belongs in $\Lambda$ or not depends only on $z$ and $\omega_{n}, n>0$, and that we may assume $(\tau \underline{\omega}, z) \in \Lambda$, otherwise $v^{x, z}\{\omega:(\underline{\omega}, x) \in \Gamma\}=0$. This guarantees that $W_{\alpha}^{j}\left(\Lambda_{\tau \omega} \cap B(z, \delta)\right)$ is well behaved. Let $T_{\tau \omega, z}=\exp _{z} F^{j}(\tau \underline{\omega}, z), \pi_{\tau \omega, z}=$ projection along $W_{\alpha}^{j}$ leaves onto $T_{\tau \omega, z}$ and $d^{T}=$ distance along $T_{\tau \omega, z}$. (Note also that these objects do not depend on $\omega$.) Since all the $\omega$ 's considered have the property that $\left|D f_{\omega}\right| \leqq C$, we have for all relevant $(\omega, y)$,

and

$$
d^{T}\left(z, \pi_{\tau \underline{\underline{\omega}, z}} f_{\omega} y\right) \leqq C d^{\hat{T}}\left(x, \hat{\pi}_{\underline{\omega}, x} y\right) \leqq C r,
$$

$$
d\left(z, f_{\omega} y\right) \leqq C d(x, y)
$$

so that in particular $f_{\omega} y \in \Lambda_{\tau \omega} \cap B(z, \delta)$.

We now define a set $A_{r, y}$ such that all $(\omega, y)$ of interest to us have the property that $f_{\omega} y \in A_{r, y}$. For $w \in M$ near $z$, let $P(w)$ denote the $d_{j}$-dimensional disk containing $w$ so that $\exp _{z}^{-1} P(w)$ is parallel to $F^{j}(\tau \underline{\omega}, z)$ in $T_{z} M$. Then, 
And so

$$
\begin{aligned}
A_{r, y}= & \left\{w \in W_{\alpha}^{j}\left(\Lambda_{\tau \omega} \cap B(z, \delta)\right): d^{T}\left(\pi_{\tau \underline{\omega}, z} w, z\right) \leqq C r\right. \\
& \text { and } \left.\operatorname{dist}\left(F^{j}(\tau \underline{\omega}, z), \exp _{z}^{-1} P(w)\right) \leqq 2 C d(x, y)\right\}
\end{aligned}
$$

$$
a_{r} \leqq \int_{B(x, \delta / C)} v^{x, z}\left\{\omega: f_{\omega} y \in A_{r, y}\right\} d \mu_{\underline{\omega}}(y)=\int_{B(x, \delta / C)}\left(\int_{A_{r, y}} \rho_{y}^{x, z} d \lambda\right) d \mu_{\underline{\omega}}(y),
$$

where $\lambda$ is the Lebesgue measure on $M$.

Suppose we let $\lambda_{P(w)}$ denote the Lebesgue measure on $P(w)$. Then using the absolute continuity of the $W^{j}$-foliation (property (v) of (2.1)) we see that for all $w$ in question

Thus we have

$$
\lambda_{P(w)}\left(A_{r, y} \cap P(w)\right) \leqq E_{0} \lambda_{T_{r \omega, z}}\left(A_{r, y} \cap T_{\tau \underline{\underline{\omega}, z}}\right)
$$

$$
\begin{aligned}
\int_{A_{r, y}} \rho_{y}^{x, z} d \lambda & \leqq \lambda\left(A_{r, y}\right)\left|\rho_{y}^{x, z}\right| \\
& \leqq Q E_{0}(C r)^{d_{J}}(2 C d(x, y))^{\left(\operatorname{dim} M-d_{J}\right)} \frac{1}{d(x, y)^{(\operatorname{dim} M+\xi)}} \\
& \leqq Q^{\prime} \frac{r^{d_{j}}}{d(x, y)^{d_{J}+\xi}}
\end{aligned}
$$

where $Q$ and $Q^{\prime}$ are constants independent of $r$.

To complete the proof, we integrate in $y$. Let $D_{n}=B\left(x, e^{-n} \delta / C\right) \backslash B\left(x, e^{-(n+1)} \delta / C\right)$. Then, if $\underline{\sigma}-2 \xi>d_{j}$, we have

$$
\begin{aligned}
\frac{a_{r}}{r^{d_{j}}} & \leqq \frac{1}{r^{d_{j}}} \sum_{n=0}^{\infty} \mu_{\omega}\left(D_{n}\right) \sup _{y \in D_{n}} \int_{A_{r, y}} \rho_{y}^{x, z} d \lambda \\
& \leqq \frac{1}{r^{d_{j}}} \sum_{n=0}^{\infty} C\left(e^{-n} \delta / C\right)^{\underline{\sigma}-\xi} \frac{Q^{\prime} r^{d_{j}}}{e^{-(n+1)\left(d_{j}+\xi\right)}} \\
& \leqq Q^{\prime \prime} \sum_{n=0}^{\infty} e^{-n\left(\underline{\sigma}-d_{j}-2 \xi\right)}
\end{aligned}
$$

where $Q^{\prime \prime}$ is independent of $r$. For the case $\underline{\sigma}-2 \xi<d_{j}$, do as above except to use the less extravagant estimate of

$$
v^{x, z}\left\{\omega: f_{\omega} y \in A_{r, y}\right\} \leqq 1 \text { for } y \in D_{n} \text { with } e^{-n}<r .
$$

(3.4) Proof of Theorem $B^{\prime}$. It suffices to show that there are measurable sets $\Delta \subset \Omega^{\mathscr{Z}} \times M$ of arbitrarily large $\mu^{*}$-measure such that for $\mu^{*}$-a.e. $(\underline{\omega}, x) \in \Delta$, there exists a continuous family of $W^{j}$-disks $\left\{D_{\gamma}\right\}$ with $\cup D_{\gamma}$ containing a neighborhood of $x$ in $\Delta_{\omega}$, and a transversal $\sigma$ so that $\mu_{\omega} \mid\left(\cup D_{\gamma} \cap \Delta_{\omega}\right)$ projected into $\sigma$ is absolutely continuous with respect to Lebesgue. Once this is established we can appeal to the absolute continuity of the $W^{j}$ foliation to show that $\left\{\mu_{\omega} \mid \Delta_{\omega}\right\}$ has absolutely continuous transverse measures as defined in (1.4).

To proof this first statement amounts to observing that the proof in (3.3) works for points in entire neighborhoods of $\Gamma_{\omega}$ using a common transversal. More precisely, fix $\left\{\omega_{n}, n \neq 0\right\}$, and $z_{0} \in M$ such that $\left(\tau \underline{\omega}, z_{0}\right) \in \Lambda$. Let $S=\exp _{z_{0}} F^{j}\left(\tau \underline{\omega}, z_{0}\right)$. 
For each $\omega \in \Omega$, let $\tilde{S}_{\omega}=f_{\omega}^{-1} S$ and $\tilde{\pi}_{\omega}=$ the projection along $W_{\alpha}^{j}$ onto $\tilde{S}_{\omega}$. Now consider $(\omega, x) \in \Gamma$ such that $f_{\omega} x \in B\left(z_{0}, \delta / 2\right)$ and define

$$
\mu^{\tilde{S}_{\omega}}(\underline{\omega}, x, r)=\mu_{\underline{\omega}}\left\{y \in \Gamma_{\underline{\omega}}: y \in B(x, \delta / 2 C) \text { and } d^{\tilde{S}}\left(\tilde{\pi}_{\underline{\omega}} x, \tilde{\pi}_{\underline{\omega}} y\right) \leqq r\right\} .
$$

The same argument as in (3.3) shows that for $\mu_{\omega}$-a.e. fixed $x$, and $v$-a.e. $\omega$ such that $f_{\omega} x \in B\left(z_{0}, \delta / 2\right)$.

$$
\liminf _{r \rightarrow 0} \frac{\mu^{\tilde{s}_{\omega}}(\underline{\omega}, x, r)}{r^{d_{j}}}<+\infty .
$$

This implies that for $\mu^{*}$-a.e. $(\underline{\omega}, x) \in \Gamma \cap F^{-1}\left(\Omega^{\mathscr{Z}} \times B\left(z_{0}, \delta / 2\right)\right)$, there is a continuous family of $W^{j}$-disks $\left\{D_{\gamma}\right\}$ with $x \in \cup D_{\gamma} \subset M_{\omega}$ such that the transverse measure is absolutely continuous with respect to Lebesgue.

\section{Proof of Version $A$ of the Results}

As with result $B$ we first give the idea of the proof in (4.1) without attempting to be precise. The formal proof is then carried out in (4.2) with some technical details postponed to (4.3).

(4.1) Idea of Proof of Theorem A. Let $\pi_{\omega, \lambda}^{j}$ again denote projection along $W_{\alpha}^{j}$ onto $\exp _{x} F^{j}(\underline{\omega}, x)$. We would like to choose a good set $\Sigma$ of positive $\mu^{*}$-measure, fix $\delta>0$, and for each $(\underline{\omega}, x) \in \Sigma$ estimate $\mu_{\underline{\omega}}\left\{y \in \Sigma_{\omega}: d(x, y) \leqq \delta, d\left(\pi_{\underline{\omega}, x}^{j} y, x\right) \leqq r\right\}$ as $r$ goes to zero as we did in Sect. 3. However, because Hypothesis A only guarantees a density for $\underline{\omega} \rightarrow E^{j}(\underline{\omega}, y), j=L, L+1$, and the $W^{j}$ manifolds are (probably) curved, we are only able to estimate the $\mu_{\omega}$-measure of

$$
\Sigma_{\underline{\omega}}^{j}(x, r, t)=:\left\{y \in \Sigma_{\underline{\omega}}: d(x, y) \leqq r^{t}, d\left(\pi_{\underline{\omega}, x}^{j} y, x\right) \leqq r\right\}
$$

for $t \in(1 / 2,1)$. Now if the transverse dimension of $\mu_{\omega}$ with respect to $W^{j}$ (which we do not claim to define) is as large as possible, then adding transverse and fiber dimensions as in (3.1) should give us

$$
\left\{\begin{array}{l}
\sigma \leqq d_{j} \Rightarrow \limsup _{r \rightarrow 0} \frac{\log \mu_{\omega} \Sigma_{\omega}^{j}(x, r, t)}{\log r} \geqq \sigma \\
\sigma>d_{j} \Rightarrow \limsup _{r \rightarrow 0} \frac{\log \mu_{\underline{\omega}} \Sigma_{\underline{\omega}}^{j}(x, r, t)}{\log r} \geqq d_{j}+t\left(\sigma-d_{j}\right)
\end{array}\right.
$$

for $j=L, L+1$. This is what we will show. The proof is sort of a nonlinear version of part of [Mat] and depends heavily on Hypothesis A.

Recall that $L$ is the smallest integer $j$ so that $\sum_{i \leqq j} \lambda_{i} m_{i}<0$, so that our goal is to show that $\gamma_{j}=m_{j}$ for $j<L$ and $\gamma_{j}=0$ for $j>L$. It is easy to see that to force this, it suffices to show

$$
\limsup _{r \rightarrow 0} \frac{\log \mu_{\underline{\omega}} \Sigma_{\omega}^{j}(x, r, t)}{\log r} \leqq\left(\sum_{i<j} \gamma_{i}\right)+t\left(\sum_{l \geqq j} \gamma_{i}\right)
$$

for $j=L, L+1$. We will show that this is a general fact, not having anything to do with random maps and valid for all $j$ with $\lambda_{j}<0$-as long as $t>1-\beta$, where 
$\beta$ is the Hölder exponent of $E^{j}$ on $\Lambda_{\underline{\omega}}$.

(4.2) Proof of Theorem A. For $\mu$-a.e. $x$ and $j=L, L+1$, let $\rho_{x}^{j}$ be the density with respect to Lebesgue of the distribution of $\underline{\omega} \rightarrow E^{j}(\underline{\omega}, x)$ in the space $\mathscr{E}_{x}$ of $\sum m_{i}$-dimensional planes in $T_{x} M$. We first make precise assertion $(*)$ in (4.1).

Let $j=L$ or $L+1$ be fixed for a while and let $\xi$ be an arbitrarily small constant. We choose $E$ and $\theta>0$ with $\theta \leqq \alpha / 100 \delta_{0}$ so that

$$
\Sigma=:\left\{(\underline{\omega}, x) \in \Lambda: \rho_{x}^{j} \leqq E \text { and } \mu_{\underline{\omega}} B(x, r) \leqq E r^{(\underline{\sigma}-\xi)} \forall r \leqq \theta\right\}
$$

has positive $\mu^{*}$-measure. Let $\pi_{\omega, x}^{j}$ be the projection along $W_{\alpha}^{j}$ into $\exp _{x} F^{j}(\underline{\omega}, x)$ as before. For $(\underline{\omega}, x) \in \Gamma$ and $t \in(\overline{0}, 1)$, we define

$$
\Sigma_{\underline{\omega}}^{j}(x, r, t)=\left\{y \in \Sigma_{\underline{\omega}}: d(x, y) \leqq r^{t} \text { and } d\left(x, \pi_{\omega, x}^{j} y\right) \leqq r\right\} .
$$

Proposition 4.2.1 (Main Proposition). Let $t \in(1 / 2,1)$. For $\mu^{*}$ a.e. $(\underline{\omega}, x) \in \Sigma$, if $\underline{\sigma}-\xi>d_{j}$, then

$$
\limsup _{r \rightarrow 0} \frac{\log \mu_{\omega} \Sigma_{\underline{\omega}}^{j}(x, r, t)}{\log r} \geqq d_{j}+t\left(\underline{\sigma}-d_{j}-\xi\right),
$$

and if $\underline{\sigma}-\xi<d_{j}$, then

$$
\limsup _{r \rightarrow 0} \frac{\log \mu_{\underline{\omega}} \Sigma_{\omega}^{j}(x, r, t)}{\log r} \geqq \underline{\sigma}-\xi .
$$

Proof of Proposition 4.2.1. We fix $\left\{\omega_{n}, n<0\right\}$ and $x \in M$. For any sequence $\underline{\omega}^{+}=\omega_{0} \omega_{1} \omega_{2} \cdots$ we will write $\underline{\omega}$ for the corresponding bi-infinite sequence. Let

$$
b_{r}=\int_{\left\{\underline{\omega} \underline{\omega}^{+}(\underline{\omega}, x) \in \Sigma\right\}} \mu_{\underline{\omega}} \Sigma_{\underline{\omega}}^{j}(x, r, t) d v\left(\omega_{0}\right) d v\left(\omega_{1}\right) \cdots .
$$

As before we will show that for $v^{v^{*}}$-a.e. $\underline{\omega}^{+}$,

$$
\left\{\begin{array}{l}
\limsup _{r \rightarrow 0} \frac{b_{r}}{r^{d_{j}+t\left(\underline{\underline{g}}-d_{j}-\xi\right)}}<+\infty \text { if } \underline{\sigma}-\xi>d_{j} \\
\text { and } \\
\limsup _{r \rightarrow 0} \frac{b_{r}}{r^{\underline{\sigma}-\xi}}<+\infty \quad \text { if } \quad \underline{\sigma}-\xi<d_{j},
\end{array}\right.
$$

which will then imply the proposition. Again we change the order of integration in $b_{r}$ to get

$$
b_{r}=\int_{B\left(x, r^{t}\right)} v^{\gamma}\left\{\underline{\omega}^{+}:(\underline{\omega}, x)(\underline{\omega}, y) \in \Sigma, d\left(x, \pi_{\underline{\omega}, x}^{j} y\right) \leqq r\right\} d \mu_{\underline{\omega}}(y) .
$$

For $y \in \Lambda_{\omega}$ near $x$, let $\tilde{\pi}_{\omega, x}^{j}(y)$ denote the unique point of intersection of $\exp _{y} E^{j}(\underline{\omega}, y)$ and $\exp _{x} F^{j}(\underline{\omega}, x)$.

Lemma 4.2.2. There is $r_{0}>0$ such that for all $x, y \in \Lambda_{\omega}$, if $d(x, y) \leqq r_{0}$, then

$$
d\left(\tilde{\pi}_{\omega, x}^{j} y, \pi_{\omega, x}^{j} y\right) \leqq C_{0} d(x, y)^{2} .
$$

We will not prove this lemma here, but note that it allows us to estimate $b_{r}$ 
for $r^{t} \leqq r_{0}$ by:

$$
b_{r} \leqq \int_{B\left(x, r^{t}\right)} v^{\mathcal{V}}\left\{\underline{\omega}^{+}:(\underline{\omega}, x)(\underline{\omega}, y) \in \Sigma, d\left(x, \tilde{\pi}_{\underline{\omega}, x}^{j} y\right) \leqq\left(C_{0}+1\right) r\right\} d \mu_{\underline{\omega}}(y) .
$$

Here we have used the fact that $\left(r^{t}\right)^{2} \leqq r$. Let

$$
\mathscr{E}_{x, y}=:\left\{e: e \in \mathscr{E}_{y}, d\left(\exp _{y}^{-1} x, e\right) \leqq 2\left(C_{0}+1\right) r\right\} .
$$

Note that we may assume that $\rho_{y}^{j}$ is defined. Then,

$$
\begin{aligned}
\left\{\underline{\omega}^{+}:(\underline{\omega}, x),(\underline{\omega}, y)\right. & \left.\in \Sigma, d\left(x, \tilde{\pi}_{\underline{\omega}, x}^{j} y\right) \leqq\left(C_{0}+1\right) r\right\} \\
& \subset\left\{\underline{\omega}^{+}: \rho_{y}^{j}\left(E^{j}(\underline{\omega}, y)\right) \leqq E \text { and } E^{j}(\underline{\omega}, y) \in \mathscr{E}_{x, y}\right\}
\end{aligned}
$$

Now, by lemma 2.6 of [Mat] we have

$$
\operatorname{Leb} \mathscr{E}_{x, y} \leqq Q \frac{r^{d_{j}}}{(d(x, y))^{d_{j}}}
$$

for some constant $Q$. So,

$$
v^{\mathscr{N}}\left\{\underline{\omega}^{+}: \rho_{y}^{j} \leqq E \text { and } E^{j}(\underline{\omega}, y) \in \mathscr{E}_{x, y}\right\} \leqq E Q \frac{r^{d_{j}}}{d(x, y)^{d_{j}}} .
$$

Letting $y$ vary in $B\left(x, r^{t}\right)$ and integrating as we did in (3.3) we get for $r^{t} \leqq \theta, r_{0}$ :

$$
b_{r} \leqq Q^{\prime} \cdot \sum_{n \geqq-t \log r} e^{-n(\underline{\sigma}-\xi)} \cdot \min \left(\frac{r^{d_{J}}}{e^{-n d_{j}}}, 1\right)
$$

which completes the proof of the main proposition.

Turning to $(* *)$ in 4.1 , we now allow $j$ to be anything with $\lambda_{j}<0$ and recall that $\beta$ is the Hölder exponent of $E^{j}$ as defined in (2.1). Let $\eta$ be a measurable partition of $\Omega^{\mathscr{Z}} \times M$ subordinate to $W^{j}$, and let $\left\{\mu_{\omega, x}^{\eta}\right\}$ be a family of conditional probability measures associated with $\eta$. We shrink $\Sigma$ in two stages. First we choose $\theta^{\prime}>0$ so that the set

$$
\begin{aligned}
\Sigma^{\prime}=:\left\{(\underline{\omega}, x) \in \Sigma: \mu_{\underline{\omega}}\left(B(x, r) \cap \Sigma_{\underline{\omega}}\right) \geqq E^{-1} r^{\bar{\sigma}+\xi}\right. \\
\left.\quad \text { and } \mu_{\underline{\omega}, x}^{j} B_{\underline{\omega}}^{j}(x, r) \leqq E r^{\left(\sum_{i \geqq j} \gamma_{i}-\xi\right)} \text { for all } r \leqq \theta^{\prime}\right\}
\end{aligned}
$$

has positive $\mu^{*}$-measure. Then we choose $\theta^{\prime \prime}>0$ so that

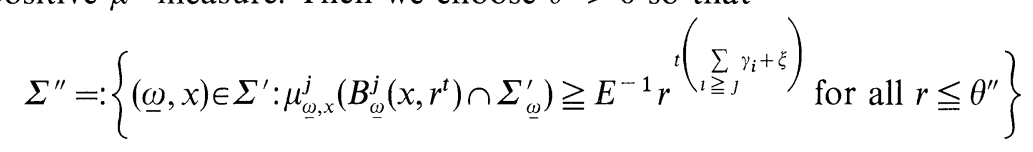

continues to satisfy $\mu^{*} \Sigma^{\prime \prime}>0$.

Proposition 4.2.3. Let $t>1-\beta$. Then for $\mu^{*}$ a.e. $(\underline{\omega}, x) \varepsilon \Sigma^{\prime \prime}$,

$$
\limsup _{r \rightarrow 0} \frac{\log \mu_{\omega} \Sigma_{\omega}^{j}(x, r, t)}{\log r} \leqq \bar{\sigma}-(1-t) \sum_{i \geqq j} \gamma_{i}+3 \xi \text {. }
$$


Proof. Let $(\omega, x) \varepsilon \Sigma^{\prime \prime}$ and consider $r<\theta, \theta^{\prime}, \theta^{\prime \prime}$ small enough that $B_{\omega}^{j}\left(x, r^{t}\right) \subset \eta_{\omega}(x)$.

We can choose $y_{1}, \ldots, y_{N_{r}} \in B_{\omega}^{j}\left(x,(r / 2)^{t}\right) \cap \Sigma_{\omega}^{\prime}$ such that:

and

$$
\text { 1. } B_{\underline{\omega}}^{j}\left(y_{i}, \frac{r}{2}\right) \text { and } B_{\underline{\omega}}^{j}\left(y_{k}, \frac{r}{2}\right) \text { are disjoint for } i \neq k
$$

$$
\text { 2. } B_{\underline{\omega}}^{j}\left(x,\left(\frac{r}{2}\right)^{t}\right) \cap \Sigma_{\omega}^{\prime} \subset \bigcup_{i=1}^{N_{r}} B_{\omega}^{j}\left(y_{i}, r\right) \text {. }
$$

Using the estimate in the definitions of $\Sigma^{\prime}$ and $\Sigma^{\prime \prime}$, we see that

$$
N_{r} \geqq \frac{E^{-1}\left(\frac{r}{2}\right)^{t\left(\sum_{l \geqq J} \gamma_{l}+\xi\right)}}{E r\left(\sum_{i \geqq j} \gamma_{t}-\xi\right)} .
$$

We have arranged for the balls $B\left(y_{i}, r / 3\right)$ to be pairwise disjoint and we will see from Lemma 4.3 .1 (stated in the next subsection) that each $B\left(y_{i}, r / 3\right) \cap \sigma_{\omega}$ is contained in $\sigma_{\underline{\omega}}^{j}(x, r, t)$. So we have

$$
\mu_{\underline{\omega}} \Sigma_{\underline{\omega}}^{j}(x, r, t) \geqq N_{r} E^{-1}(r / 3)^{\bar{\sigma}+\xi},
$$

which is the desired estimate.

Thus, by fixing $t$ with $1 / 2,1-\beta<t<1$, comparing the conclusions of Proposition 4.2.1 and 4.2 .3 for $j=L, L+1$ and letting $\xi$ go to zero, we complete the proof of Theorem A.

\section{(4.3) Proof of Remaining Estimates}

Lemma 4.3.1. There exists $r_{1}>0$ such that for all $x, y, z$ in $\Lambda_{\omega}$ and $r \leqq r_{1}$, if $y \in B_{\underline{\omega}}^{j}\left(x, r^{t}\right)$ and $d(y, z) \leqq r / 3$, then

$$
d\left(x, \pi_{\underline{\omega}, x}^{j} z\right) \leqq r .
$$

We first write down the underlying estimates, which we state without proof in the following sublemma:

Sublemma (a) Suppose $\phi: \mathscr{R} \rightarrow \mathscr{R}^{+}$satisfies $\phi^{\prime} \leqq 2 D_{0} \phi^{\beta}$.

Then for $s \geqq 0$,

$$
\phi(s) \leqq\left[(1-\beta) 2 D_{0} s+\phi(0)^{1-\beta}\right]^{1 / 1-\beta} .
$$

(b) If, in addition we have $t>1-\beta, r$ sufficiently small, $\phi(0) \leqq 2 r / 3$ and $s \leqq r^{t}$, then

$$
\phi(s) \leqq r .
$$

Proof of Lemma 4.3.1. Let $g_{1}, g_{2}: E^{j}(\underline{\omega}, x) \rightarrow F^{j}(\underline{\omega}, x)$ be functions whose graphs are $\exp _{x}^{-1} W_{\alpha}^{j}(\underline{\omega}, y), \exp _{\alpha}^{-1} W_{\alpha}^{j}(\underline{\omega}, z)$ respectively. We need an upper bound on $\mid g_{1}(0)-$ 
$g_{2}(0) \mid$. Let $\exp _{x}^{-1} y=\left(u, g_{1}(u)\right)$ and define

$$
\phi(s)=\left|g_{2}\left(\left(1-\frac{s}{|u|}\right) u\right)-g_{1}\left(\left(1-\frac{s}{|u|}\right) u\right)\right| .
$$

We assume that $r_{1}$ is sufficiently small that $D \exp _{x}^{-1}$ in the relevant region is near the identity. Property (iv) in (2.1) tells us that

$$
\left|\frac{d \phi}{d s}\right| \leqq\left|D g_{2}\left(\left(1-\frac{s}{|u|}\right) u\right)-D g_{1}\left(\left(1-\frac{s}{|u|}\right) u\right)\right| \leqq 2 D_{0} \phi(s)^{\beta}
$$

If $r_{1}$ is small, then $g_{2}$ has a small slope, so it follows from $d(y, z) \leqq r / 3$ that

$$
\phi(0)=\left|g_{2}(u)-g_{1}(u)\right| \leqq 2 r / 3 .
$$

Also $|u| \leqq r^{t}$. Apply the sublemma to finish the argument.

Lemma 4.2.2 is proved similarly. We suggest carrying out the estimates in $T_{y} M$ via the $\exp _{y}^{-1}$ map and using property (iii) $\mathrm{b}$ iv. in (2.1). The constant $r_{0}$ should be chosen so that $\forall x, y \in \Lambda_{\underline{\omega}}$ with $d(x, y)<r_{0}, F^{j}(\underline{\omega}, x)$ and $E^{j}(\underline{\omega}, y)$ are roughly perpendicular.

\section{Proof of Theorem $\mathbf{A}^{\prime}$ and Application to Stochastic Flows}

5.1 Proof of Theorem $A^{\prime}$. Let $(x, z) \rightarrow v^{x, z}$ be defined Lebesgue a.e. as in (1.4). Take $j=L$ or $L+1$ and let $\mathscr{E}_{z}$ be the set of $\left(\sum_{j \leqq l} m_{i}\right)$ dimensional subspaces of $T_{z} M$. Observe that $E^{j}(\tau \underline{\omega}, z)$ is independent of $\omega_{0}$ and

$$
E^{j}(\underline{\omega}, x)=D f_{\omega_{0}}^{-1} E^{j}\left(\tau \underline{\omega}, f_{\omega_{0}} x\right),
$$

so that the distribution of $E^{j}(\underline{\omega}, x)$ in $\mathscr{E}_{x}$, conditioned on $f_{\omega_{0}} x=z$ and $E^{j}(\tau \underline{\omega}, z)=e$ for $e \in \mathscr{E}_{z}$ is given by:

$$
q_{x, z, e}(\Delta)=v^{x, z}\left\{\omega: D f_{\omega}^{-1} e \in \Delta\right\} .
$$

We shall prove under Hypothesis $\mathrm{A}^{\prime}$ that for Lebesgue-a.e. $(x, z)$ and all $e$ in $\mathscr{E}_{z}$, the measure $q_{x, z, e}$ is absolutely continuous with respect to Lebesgue on $\mathscr{E}_{x}$. Integrating in $z$ and $e$, we prove that Hypothesis $\mathrm{A}$ is satisfied, and thus Theorem $\mathrm{A}^{\prime}$ follows.

Consider a measurable family $(x, z) \rightarrow \tilde{v}^{x, z}$ of probability measures on $\Omega$ with the property that for Lebesgue a.e. fixed $z,\left\{\tilde{v}^{x, z}, x \in M\right\}$ is a system of conditional measures associated with the partition ${ }_{z} P$ on $\Omega$ defined by

$$
{ }_{z} P(x)=\left\{\omega: f_{\omega}^{-1} z=x\right\} .
$$

Then, the measures $v^{x, z}$ and $\tilde{v}^{x, z}$ are carried by the same set $P_{x}(z)={ }_{z} P(x)$, and it is easy to verify that they are in fact equivalent for Lebesgue-a.e. $(x, z)$.

Now, for Lebesgue-a.e. $z$ and every $e$ in $\mathscr{E}_{z}$, Hypothesis $\mathrm{A}^{\prime}$ tells us that for Lebesgue. a.e. $x$, the measure $\tilde{q}_{x, z, e}$ is absolutely continuous with respect to Lebesgue 
on $\mathscr{E}_{x}$, where

$$
\tilde{q}_{x, z, e}(\Delta)=\tilde{v}^{x, z}\left\{\omega: D f_{\omega}^{-1} e \in \Delta\right\} .
$$

Since $\tilde{q}_{x, z, e}$ and $q_{x, z, e}$ are image measures of $\tilde{v}^{x, z}$ and $v^{x, z}$ respectively under $\omega \rightarrow D f_{\omega}^{-1} e$, the measure $q_{x, z, e}$ is also absolutely continuous with respect to Lebesgue on $\mathscr{E}_{x}$.

5.2 Stochastic Flows. We shall discuss Hypothesis $\mathrm{A}^{\prime}$ in the case of the stochastic flow arising from a stochastic differential equation. Let $X_{k}, k=0,1, \ldots, m$, be $(m+1) C^{\infty}$-vector fields on a compact manifold $M$ and $B^{k}, k=1, \ldots, m$, be $m$ independent real Brownian motions defined on a probability space $\bar{\Omega}$. We write

$$
d \xi_{t}=X_{0} d t+\sum_{k=1}^{m} X_{0} \circ d B_{t}^{k}
$$

for the Stratonovich stochastic differential equation associated with the $X_{i}$ 's. Then there exists a map

$$
\phi: \bar{\Omega} \rightarrow C\left(\mathscr{R}_{+}, \operatorname{Diff}^{\infty} M\right)
$$

such that for every fixed $t_{0}>0$, we have

i) $\phi_{0}=$ identity,

and ii) the "increments" $\phi_{(n+1) t_{0}} \phi_{n t_{0}}^{-1}$ are i.i.d. in $\operatorname{Diff}^{\infty}(M)$,

and such that $\xi_{t}(\omega)=\phi_{t}(\omega) x$ is the solution of $(*)$ with $\xi_{0}(\omega)=x$ (see e.g. $[\mathrm{Ku}]$ ).

Asymptotic properties of the stochastic flow $\phi$ are the same as those of the system $\mathscr{X}(M, v)$, where $v$ is the distribution of $\phi_{t_{0}}$. Here, $\mathscr{X}(M, v)$ satisfies the condition in (1.1) (see [Ki] and [Ki2]).

Let $G L(M)$ denote the linear frame bundle of $M$. Given any vector field $X$ on $M$, we define a vector field $\tilde{X}$ on $G L(M)$ as follows: let $\psi_{t}$ be the flow generated by $X$. Then for $F$ in $C^{\infty}(G L(M), \mathscr{R})$ we define $\tilde{X} F$ by

$$
\tilde{X} F(x, e)=\left.\frac{d}{d t} F\left(\psi_{t} x, D\left(\psi_{t}\right)_{x} e\right)\right|_{t=0} .
$$

Consider the Strantonovich stochastic differential equation on $G L(M)$ defined by

$$
d \tilde{\xi}_{t}=\tilde{X}_{0} d t+\sum_{k=1}^{m} \tilde{X}_{k}^{\circ} d B_{t}^{k}
$$

Observe that if $\tilde{\phi}: \bar{\Omega} \rightarrow C\left(\mathscr{R}_{+}, \operatorname{Diff}^{\infty}(G L(M))\right.$ is the stochastic flow defined by (**), then for $(x, e) \in G L(M), \omega \in \bar{\Omega}, t_{0}>0$,

$$
\tilde{\phi}_{t_{0}}(\omega)(x, e)=\left(\phi_{t_{0}}(\omega) x, D\left(\phi_{t_{0}}(\omega)\right)_{x} e\right) \quad \text { (see e.g. [IW]). }
$$

We claim that if the operator $L$ on $C^{\infty}(G L(M))$ defined by

$$
L F=-\tilde{X}_{0} F+\sum_{k=1}^{m} \tilde{X}_{k}^{2} F
$$

is hypoelliptic, then the system $\mathscr{X}(M, v)$ defined by $(*)$ and $t_{0}>0$ satisfies Hypothesis $\mathrm{A}^{\prime}$. This implies in particular that for $m \geqq \operatorname{dim} M+(\operatorname{dim} M)^{2}$, there is an open dense subset in the space of $(m+1)$-tuples of vector fields on $M$ on which Hypothesis 
$\mathrm{A}^{\prime}$ is satisfied.

Let us elaborate a little on the claim above. If $\left\{\hat{B}_{t}^{h}, 0 \leqq t \leqq t_{0}, k=1, \ldots, 1, m\right\}$ denotes the backward Brownian motions, then the backward equation associated with $(*)$ is

$$
d \eta_{t}=-X_{0}\left(\eta_{t}\right) d t+\sum_{k=1}^{m} X_{k}\left(\eta_{t}\right) \circ d \hat{B}_{t}^{k} 0 \leqq t \leqq t_{0} .
$$

Let $\hat{\phi}: \bar{\Omega} \rightarrow C\left(\left[0, t_{0}\right] \times \operatorname{Diff}^{\infty}(M)\right)$ be the corresponding stochastic flow. Then the distribution of $\phi_{t_{0}}^{-1}(\omega)$ and of $\hat{\phi}_{t_{0}}(\omega)$ in $\operatorname{Diff}^{\infty}(M)$ are the same (see $[\mathrm{Ku}]$ ).

Proceeding analogously with $(* *)$ to obtain the backward equation $(\widehat{* *})$, we see that for any $(y, e)$ in $G L(M)$, the distribution of $\left(\phi_{t}^{-1}(\omega) y, D\left(\phi_{t}^{-1}(\omega)\right)_{y} e\right)$ is given by the fundamental solution of the diffusion equation associated with $(\widehat{* *})$.

The hypoellipticity condition implies that for all $(y, e)$ in $G L(M)$ and a fixed $t_{0}>0$, the distribution of $\left(\phi_{t_{0}}^{-1} y, D\left(\phi_{t_{0}}^{-1}\right)_{y} e\right)$ is absolutely continuous with respect to Lebesgue on $G L(M)$. Hypothesis $\mathrm{A}^{\prime}$ follows since for each $k, 1 \leqq k \leqq m$, the projection from $G L(M)$ to $\operatorname{Gr}(M, k)$ preserves the Lebesgue measure class.

Acknowledgements. We are grateful to a number of friends for their comments and suggestions, in particular to J. Palis and R. Mañé for giving us references [Mar] and [Mat] which provide the key ideas behind this work.

\section{References}

[BK] Brin, M., Kifer, Y.: Dynamics of Markov chains and stable manifolds for random diffeomorphisms. Preprint

[BN] Brin, M., Nitecki, Z.: Absolute continuity of stable foliation in Hilbert space. In preparation

[C] Carverhill, A.: Flows of stochastic dynamical systems: Ergodic theory. Stochastic 14, 273-317 (1985)

[ER] Eckmann, J.-P., Ruelle, D.: Ergodic theory of chaos and strange attractors. Rev. Mod. Phys. 57, 617-656 (1985)

[FKYY] Frederickson, P., Kaplan, J. L., Yorke, E. D., Yorke, J. A.: The Lyapunov dimension of strange attractors. J. Differ. Equations 49, 183-207 (1983)

[IW] Ikeda, N., Watanabe, S.: Stochastic differential equations and diffusion processes. Amsterdam: North-Holland Kodansha 1981

[Ki1] Kifer, Y.: Ergodic theory of Random Transformations. Progress in Probability and Statistics. Boston: Birkhäuser 1986

[Ki2] Kifer, Y.: A note on integrability of $C^{r}$-norms of stochastic flows and applications, 1987 preprint

[KS] Katok, A., Strelcyn, J.-M.: Smooth maps with singularities; invariant manifolds, entropy and billiards. Lecture Notes in Math., Vol. 1222, Berlin, Heidelberg, New York: Springer 1986

$[\mathrm{Ku}]$ Kunita, H.: Stochastic differential equations and stochastic flow of diffeomorphisms. Ecole d'Eté de Probabilités de Saint-Flour XII. 1982; Hennequin, P.-L. (ed.). Lecture Notes in Math., Vol. 1097. Berlin, Heidelberg, New York: Springer 1984

[Le] Le Jan, Y.: On isotropic Brownian motions. Z. Wahrscheinlichkeitstheorie Verw. Geb. 70, 609-620 (1985)

[LY1] Ledrappier, F., Young, L.-S.: The metric entropy of diffeomorphisms, part II: Relations between entropy, exponents and dimension. Ann. Math. 122, 540-574 (1985)

[LY2] Ledrappier, F., Young, L.-S.: Entropy formula for random transformations. Preprint.

[Man] Mañe, R.: On the dimension of the compact invariant sets of certain nonlinear maps. In: Dynamical systems and turbulence, Warwick, 1980. Lecture Notes in Mathematics, Vol. 898. pp. 230-242 Berlin, Heidelberg, New York: Sprınger 1981 
[Mar] Marstrand, J. M.: Some fundamental geometrical properties of plane sets of fractional dimensions. Proc. Lond. Math. Soc. 4, 257-302 (1954)

[Mat] Mattila, P.: Hausdorff Dimension, Orthogonal projections and Intersections with planes. Ann. Acad. Sci. Fennicae, Series A1 Math. 1, 227-244 (1975)

[T] Thieullen, P.: Fibrés dynamiques asymptotiquement compacts: Exposants de Lyapunov. Entropie. Dimension. Ann. Inst. Henri Poincaré Analyse non linéaire 4, 49-97 (1987)

[Y] Young, L.-S.: Dimension, entropy and Lyapunov exponents. Erg. Theory Dynam. Sys. 2 , 109-124 (1982)

Communicated by J.-P. Eckmann

Received May 11, 1987 